\title{
A three-step methodology to improve domestic energy efficiency
}

\author{
Albert Molderink, Student member, IEEE, Vincent Bakker, Student member, IEEE, Maurice G.C. Bosman, Johann \\ L. Hurink, Gerard J.M. Smit
}

\begin{abstract}
Increasing energy prices and the greenhouse effect lead to more awareness of energy efficiency of electricity supply. During the last years, a lot of technologies have been developed to improve this efficiency. Next to large scale technologies such as windturbine parks, domestic technologies are developed. These domestic technologies can be divided in 1) Distributed Generation (DG), 2) Energy Storage and 3) Demand Side Load Management. Control algorithms optimizing a combination of these techniques can raise the energy reduction potential of the individual techniques. In this paper an overview of current research is given and a general concept is deducted. Based on this concept, a three-step optimization methodology is proposed using 1) offline local prediction, 2) offline global planning and 3) online local scheduling. The paper ends with results of simulations and field tests showing that the methodology is promising.
\end{abstract}

Keywords: Micro-generation, Energy efficiency, Microgrid, Virtual Power Plant, Smart grid

\section{INTRODUCTION}

Due to increasing energy prices and the greenhouse effect more efficient electricity supply is desirable, preferably based on renewable sources. In the last years, a lot of technologies have been developed to improve the efficiency of the electricity usage and supply. One of the most eye catching technologies is generation based on renewable sources like large windturbine and photovoltaic (PV) parks. Also on domestic level a lot of technologies are under development. These technologies range from $\mathrm{PV}$ on roofs and micro Combined Heat and Power (microCHP) [1] up to controllable appliances [2]. The technologies can be subdivided in three groups:

- Distributed Generation (DG) In contrast to electricity generation in a few large power plants, in the future a growing share of the electricity is generated in smaller, geographically distributed generators. DG often has a higher efficiency or is based on (uncontrollable) renewable sources. Furthermore, DG lowers transportation costs due to on-site production. The DG generators range from windparks and bio-fuel plants on a megawatt level to domestic generators. Domestic generators are generators on a kilowatt level placed in or nearby houses. These generators can produce heat and/or electricity.

- Distributed Electricity Storage Especially with a growing amount of renewable sources in the electricity supply chain there is a growing demand for electricity storage

This research is conducted within the Islanded House project supported by E.ON Engineering and the SFEER project supported by Essent, Gasterra and STW.

All authors are with University of Twente, Department of Computer Science, Mathematics and Electrical Engineering, P.O. Box 217, $7500 \mathrm{AE}$ Enschede, The Netherlands,a.molderink@utwente.nl
[3]. Electricity may be produced more efficiently at certain time periods (e.g. at night) or only at certain time periods (e.g. depending on available wind or sun) and thus may need to be stored. Large capacity electricity storage is difficult and has high losses, so distributed electricity storage may be a solution. A large scale introduction of electrical cars adds a large storage capacity since cars are typically only used a couple of hours per day.

- Demand Side Load Management Just as electricity storage, demand side load management can increase the generation efficiency by peak shaving [4] and by shifting load to more beneficial periods [5]. Parts of an appliance (e.g. the heating element of a dryer) can be temporarily switched off or the start of an appliance can be postponed [2]. About $50 \%$ of the load in houses is dedicated to refrigerators, freezers, heaters, washing machines and dryers [6]. These appliances can be managed with only a little discomfort for the residents. Field tests in the USA have shown that optimizations with these manageable appliances already can lead to significant peak reductions [2]. Furthermore, when residents choose for a certain level of discomfort, e.g. a deviation of $0.5^{\circ} \mathrm{C}$ from the settled room temperature, even more scheduling freedom is gained. Of course there has to be an incentive for the residents to accept the reduction in comfort.

Although these three technologies already increase the energy efficiency, cooperation between the technologies and the existing infrastructure may lead to additional benefits.

In this paper a control methodology is proposed to manage the cooperation between these technologies. The methodology is based on a three-step approach to control domestic electricity and heat demand, as well as the generation and storage of heat and electricity.

To verify the quality of the methodology, a simulator has been developed and is used to simulate various scenarios. Furthermore, prototypes are built to study controllability of the devices in the real world and to implement first versions of the algorithms.

The remaining of this paper is structured as follows. The next section describes the optimization potentials and methods. Section III gives an overview of the related work and ends with a general concept based on the related work. Next, section IV describes the approach following from this general concept and proposes a three-step optimization methodology. Section $\mathrm{V}$ describes the built prototypes and section VI describes the results of simulations and prototype tests using the optimization methodology. The last section discusses these results. 


\section{OptimizATION POTENTIAL}

The goal of a control methodology is to manage the cooperation between the domestic technologies to use the maximum optimization potential. Some technologies itself may lead to a decreased domestic energy usage (electricity and heat). However, the goal of this method is not to decrease domestic energy usage but to optimize the usage to increase efficiency of energy supply(e.g. power plants) and decrease usage of fossil fuels. Next to improving efficiency, optimizing the usage of the domestic technologies can (and has to) enhance the reliability of supply [7], [8]. The objective of such a control methodology is to optimize the electricity import and export profile of (a group of) houses. The optimization objective can differ, depending on the stakeholder of the global controller. One common element in most objectives is peak shaving. The production patterns of the large power plants are given by the (residential) demand minus the distributed generation. Peaks in these production patterns are supplied by less efficient peak power plants and should therefore be lowered [9]. For the power plants an ideal production pattern is a constant production.

Fluctuations in demand are caused by the stochastic nature of demand; people switch their TV on when they like to watch TV. Furthermore, the new technologies mentioned in the previous section introduce even more fluctuations. DG based on renewable sources like sun and wind has a very fluctuating production pattern and even for a large scale introduction of microCHPs a fit-and-forget strategy is not applicable [7]. Also the introduction of electrical cars introduces not only a (storage) potential, but may introduce a threat to the stability of the grid. When a large number of cars start to charge their batteries at the same time it may cause capacity problems, thus charging should be managed and/or synchronized with (local) production. Finally, the grid is designed and built for an electricity stream from power plants to houses, the transformers can not manage large electricity flows from the low voltage to the high voltage parts of the grid. Therefore, ideally the locally produced electricity should be used locally, i.e. within the neighborhood without passing a transformer.

Next to different objectives, control methodologies can have different scopes: a local scope (within the house), a scope on a group of houses e.g. a neighborhood (microgrid) or a global scope (Virtual Power Plant). Within every scope different optimization objectives can be used.

- Local scope On a local scope the import from and export into the grid can be optimized, without cooperation with other houses. Possible optimization objectives are shifting electricity demand to more beneficial periods (e.g. nights) and peak shaving. The ultimate goal can be to create an independent house, which implies no net import from or net export into the grid. A house that is physically isolated from the grid is called an islanded house. The advantages of a local scope is that it is relatively easy, there is no communication with a global unit (privacy) and there is no external entity deciding which appliances are switched on or off (social acceptance).

- Microgrid In a microgrid a group of houses together optimize their combined import from and export into the grid, optionally combined with larger scale DG (e.g. windmills). The objectives of a microgrid can be shifting loads and shaving peaks with as ultimate goal an islanded microgrid. Advantage of a group of houses is that their joint optimization potential is higher than that of individual houses since the load profile is more flattened (e.g. startup peaks of appliances disappear in the combined load). Furthermore, multiple microgenerators working together can supply more demand than individual microgenerators since better distribution in time of the production is possible [10]. However, for a microgrid a more complex optimization methodology is required.

- Virtual Power Plant (VPP) The original VPP idea is to manage a large group of micro-generators with a total capacity comparable to a conventional power plant. Such a VPP can replace a power plant while having a higher efficiency, and moreover, it is much more flexible than a normal power plant. Especially this last point is interesting to react on fluctuations. This original idea of VPP can of course be extended to all domestic technologies. However, for a VPP also a complex optimization methodology is required. Furthermore, communication with every individual house is required and privacy and acceptance issues may be a social problem.

\section{RELATED WORK}

A lot of research about improving energy efficiency using domestic potential is going on at the moment. It is, in general, agreed that it is both desirable and necessary to manage DG and optimize efficiency. In [7] it is stated that a fit-and-forget introduction of domestic DG will cause stabilization problems. Furthermore, the large scale introduction of renewables requires a new grid design and management. A study of the International Energy Agency concludes that, although DG has higher capital costs than power plants, it has potential and that it is possible with DG to supply all demand with the same reliability, but with lower capacity margins [8]. This study foresees that the supply can change to decentralized generation in three steps: 1) accommodation in the current grid, 2) introduction of a decentralized system cooperating with the central system and 3) supplying most demand by DG. However, both [7] and [8] indicate that commercial attainability and legislation are important factors for the success of the introduction of DG.

Most research projects focus in first instance on introducing and managing (domestic) DG. In [11] the impact of DG on the stability of the grid itself is studied, i.e. whether the oscillatory stability of the grid and transformers can be improved with DG. Their conclusion is that is is possible to improve the stability when the generators are managed correctly. The authors of [12] conclude, based on UK energy demand data, that it is attractive to install microCHPs to reduce carbon dioxide emission.

Next to DG, energy storage and demand side load management are also research topics. One of the options is to combine windmills with electricity storage to level out the fluctuations by predicting the production and planning the amount of electricity exported to the grid [13]. In [14] and [15] Grid Friendly Appliances are described. These appliances switch (parts of) their load off when the frequency of the grid 
deviates too much. This frequency deviation is a measure for the stress of the grid and therefore these appliances can shift their load to periods with lower electricity demands.

A lot of control methodologies for DG, energy storage, demand side load management or a combination of these are described in literature. Most of the researchers propose agent based methodologies. These agent based methodologies propose an agent per device [16]. The agents give their price for energy production (switching an appliance off is seen as production); via a market principle it is decided which agents are allowed to produce. Since there are a lot of agents, the information is aggregated on different levels in a hierarchical way. The research described in [6] combines all three domestic technologies: demand side load management offers $50 \%$ of the potential. To reach this, there have to be incentives for the residents to allow some discomfort. Furthermore, both electricity and heat are considered and agents use predictions to determine their cost function. The PowerMatcher described in [17] and [18] also takes the network capacities into account. This methodology is rather mature; it is a product capable of being used in field tests [19]. In this field tests, a peak reduction of $30 \%$ is reached when a temperature deviation of one degree of the thermostat is allowed. To be able to reach objectives, business agents can be added that influence the biddings in the auction market. Furthermore, the authors of [20] compare the results of individual (local) and overall (global) optimizations. They conclude that global optimizations lead to better results. Next, they claim that agent based methodologies outperform non-agent based methodologies since agent based methodologies take more (domestic) information into account.

Next to agent based methodologies, there are also methodologies not based on agents. The research described in [21] proposes a method that is capable to aim for different objectives. For every device a cost function is determined for both heat and electricity. Using a Non Linear Problem definition the optimal on/off switch pattern is found. The authors of [22] address the problems of both agent and non-agent based solutions: non-agent based solution are less scalable and agent based solutions need local intelligence and are not transparent. Therefore, they propose a combination: aggregate data on multiple levels, while these levels contain some intelligence. The aggregation is done with a database, the control methodology is rule based. In [23] a methodology is proposed using Stochastic Dynamic Programming (SDP). The stochastic part of the methodology considers the uncertainty in predictions and the stochastic nature of (renewable) production and demand.

Most methodologies use some sort of prediction of demand and/or production. This can be predicted rather good with neural networks, as described in [24] and [25]. The predictions follow the trend rather good.

\section{A. General idea}

As describe above, there are many research projects investigating energy efficiency optimization. From the mentioned research, simulations and field tests it can be concluded that the efficiency can be improved significantly, especially when all three types of technologies are combined. All methodologies have split up the control into a local and a global part.
Furthermore, most methodologies use prediction to adapt to the production and demand patterns and an online algorithm deciding on device level. In general two different methods are used: 1) agent based using a market principle and 2) mathematical optimization methodologies.

The methodology proposed in this paper uses three steps and is split up into a local and a global part: 1) local offline prediction, 2) global offline planning and 3) local online scheduling. Because of the scalability, the global planning has a hierarchical structure and aggregates data and plannings on different levels (neighborhood, city, etc.). The scope of the planning and thus the global controller determines the scope (i.e. the planning can be made on a local scope). Especially the three steps and the global planning differs from the rest of methodologies described in literature. Furthermore, the methodology is not agent based and uses other mathematical optimization methods or heuristics than the methodologies described above. The global planning is based on Dynamic Programming, the local controller is based on cost functions and Integer Linear Programming (ILP).

\section{APPROACH}

Our research focuses on the development of algorithms for the control methodology. The development and verification of the algorithms is split up in three steps.

1) Algorithm development The first step is to develop the required algorithms. These algorithms are based on analysis of the current situations, objectives, possible scopes and observations of the real-world systems and data.

2) Simulations These algorithms are verified with a selfbuilt simulator [26]. This simulator can use real-world data to simulate multiple houses and verify the algorithms that decide when to start/stop the microgenerators, how much electricity flows from/to the battery, how much electricity is imported/exported from the grid and which appliances to supply. With the simulations of the algorithms the optimization results are verified, e.g. it can be simulated whether it is possible to decrease the peak imports from the grid.

3) Field tests The third step is to develop prototypes. With these prototypes it is examined whether the algorithms are also applicable in real world situations. Furthermore, it is verified whether the stated assumptions are valid.

The current developed algorithms are flexible enough to cope with different house configurations, levels of control and optimization objectives.

\section{A. Model}

The model of a single house is shown in Fig. 1. Every house consists of (several) micro-generators, heat and electricity buffers, appliances and a local controller. Multiple houses are combined into a grid, exchanging electricity and information between the houses.

Electricity can be imported from and exported to the grid. Heat is produced, stored and used only within the house. All domestic heat and electricity devices are divided into three groups: 


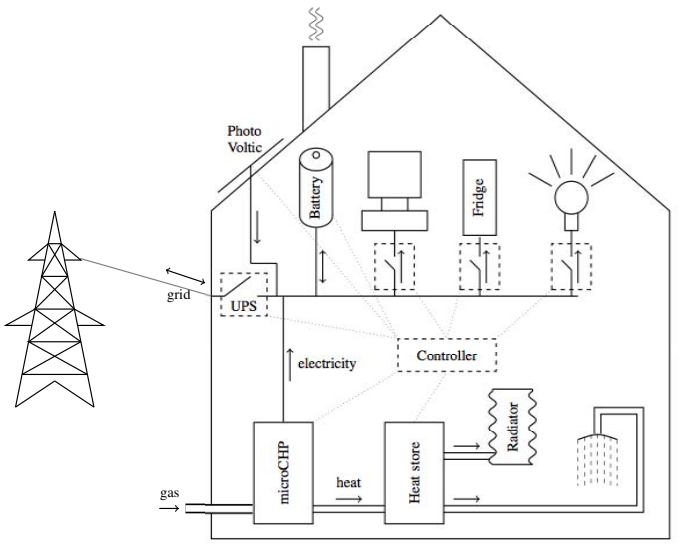

Fig. 1. Model of domestic energy streams

- Producers produce electricity and/or heat. All available micro-generators are modelled in this way, considering that the generation can be zero or even negative. A microCHP device produces electricity and heat, a Photo Voltaic produces only electricity where a conventional electric heater generates heat with a negative electricity production.

- Buffers store electricity and/or heat. When there is more energy production than consumption (and export) there is a surplus that flows into (one of) the buffers. A shortage (more consumption than production and import) flows out of (one of) the buffers.

- Consumers can consume electricity and/or heat. All consumers are modelled within this group, from fridges and coffee makers to central heating and hot tap water.

Every producer, buffer and consumer is called a device. Heat and electricity production can be coupled on device level. For example some producers produce heat and electricity at the same time, hence production of heat and electricity are coupled. A microCHP does either produce heat and electricity or nothing at all. The same holds for consuming devices, e.g. a hot fill washing machine. A more detailed description of the model can be found in [27].

Within the model, the planning horizon is discretisized resulting in a set of consecutive time intervals. The number of intervals depends on the length of the planning horizon and the length of the intervals.

\section{B. Methodology}

The goal of the local energy management methodology is to make a generic solution for different (future) domestic technologies and house configurations. Furthermore, multiple objectives are possible and the scope of the methodology can differ. That is, the methodology needs to be very flexible and generic. Since there can be global objectives (e.g. in case of a VPP) and the actual control of devices is on domestic level both a global and a local control are needed.

The primary functionality is to control the domestic generation and buffering technologies in such a way that they are used properly and the required heat and electricity supply is guaranteed for the residents. The scheduling freedom of

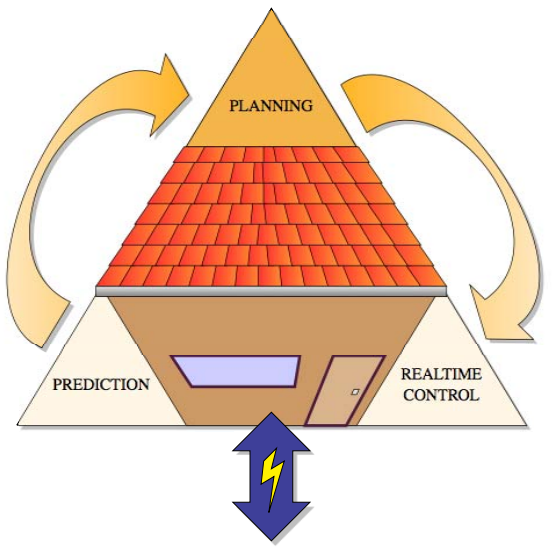

Fig. 2. Three step methododolgy

the domestic devices within these constraints can be used for optimizations. More scheduling freedom can be gained when residents are willing to decrease their comfort level. This (small) decrease in comfort should lead to some benefits for the residents, e.g. a reduced electricity bill.

Summarizing, a list of requirements for the energy management methodology is:

1) Multiple scenarios with different objectives and costs for specific devices should be possible.

2) Both a local and global controller cooperating by responding on steering signals and send status information.

3) Guaranteed comfort level chosen by the resident, given the incentives.

4) Both heat and electricity are considered and coupled to include combined heat/electricity producers and consumers.

5) Offline prediction and planning to forecast net demand on beforehand.

6) Online scheduling possibilities for instantaneous matching of supply and demand and to respond on steering signals.

7) Device-level cost functions should include present and future technologies.

The proposed management methodology is divided into three steps and there is a local (within the house) and a global (combining multiple houses) part. First, the energy demand and therefore the optimization potential for every individual house is predicted. Next, the predictions of the individual houses are aggregated and a global planning is made. In the last step, a local scheduler in every house schedules the appliances in realtime, using the global planning as an input. The basic goal of this methodology is to supply all residential heat and electricity demand without loss of comfort while optimizing the overall energy efficiency. This threestep approach is shown in Figure 2 and discussed in more detail below. The combination of prediction, local controllers and global controllers can be extended to a Smart Grid [7] solution, controlling non-domestic DG, non-domestic buffers and domestic imports/exports optimizing efficiency of central power plants.

1) Local offline prediction In the first step a prediction of the 


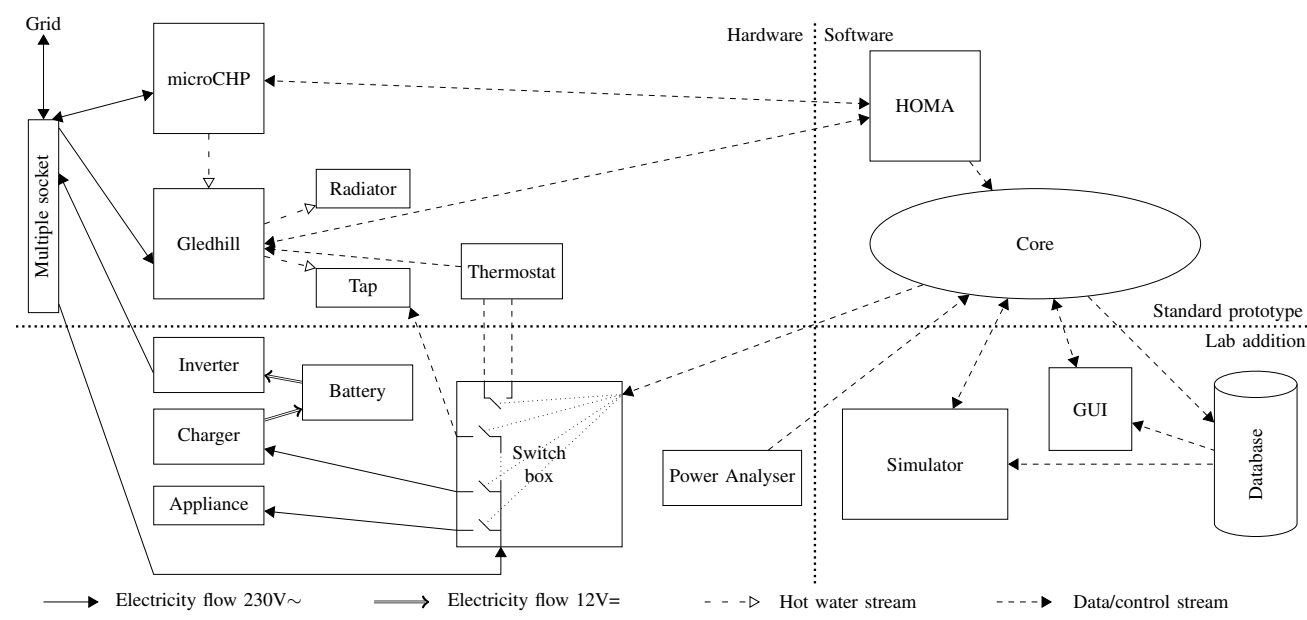

Fig. 3. Schematic overview of the hard- and software of the prototypes

energy demand and production is done for each house. This local information is necessary to decide the local production potential and scheduling freedom of the microgenerators, buffers and appliances. A neural network approach is used for this prediction [25], at the moment only the heat demand is predicted. When the scope is not local, this information is sent to a global controller for the second step.

2) Global offline planning In the second step the local potential is assigned to actual plannings, based on local (domestic) and global (VPP) objectives [28]. The planning process considering only microCHP generators is already known to be NP-complete in the strong sense [29]. Therefore, heuristics are used for the planning. These global controllers have a hierarchical structure; they aggregate the data and optionally they send it to a higher scope controller. The global controllers determine a planning based on the information they receive (from local controllers or from lower scope global controllers) and send this information back to the local controllers. At the moment the planning algorithm is tailored to heat/electricity combined generators in combination with a heat buffer. Furthermore, the global controller needs information of each house about generator characteristics and heat buffer capacity. 3) Local Realtime control The last step is a local controller for online scheduling, i.e. it decides which appliances are switched on/off, etc. Whereas the first two steps can be done offline, the devices need to be (online) controlled in realtime. In this realtime control the runs of individual microgenerators need to be (re)scheduled, if the reality differs too much from the prediction. The local controller has no knowledge of the global state but can react on steering signals (e.g. realtime pricing) from a global controller (based on the planning). The realtime control is based on cost function for every device and minimizes the total costs (where grid import also has a cost). A description of the used realtime control algorithm can be found in [30]. Also when there is no two-way communication with a global controller, it is possible to steer the import/export with steering signals (i.e. electricity price). However, since there is no knowledge of the individual houses on a global level, the result of the pricing signals is not known and no guarantees can be given.

The methodology is based on device-level cost functions described in [30]. With these cost functions residents can define their priorities and which incentive allows discomfort.

\section{PRototype}

For the field tests we have two different types of prototypes, one installation in a laboratory and multiple installations in regular houses replacing the conventional heat supply. The methodologies are first tested on the laboratory installation (a real test environment) and next they are implemented on the installations in houses.

\section{A. Hardware prototype}

The basis of the testbed is a Whispergen[31] microCHP in combination with a Gledhill[32] heatstore. The Whispergen is a Stirling engine based microCHP producing both heat and electricity. The electricity is fed back to the domestic electricity infrastructure and therefore to the grid, the heat is stored in the Gledhill. The Gledhill supplies all heat demand, both for central heating and hot water taps.

In a normal situation the boiler starts when the hot water flow starts or when the thermostat gives a signal for space heating demand. Since the heat demand is supplied by the Gledhill, an extra signal is required to signal the Whispergen when it has to produce heat (e.g. when the level in the Gledhill is low). A controller giving this signal is built in the Gledhill.

HOMA Software B.V. developed monitoring and managing software that communicates with the Whispergen and Gledhill. This software logs on a minute base the status of the installation. Furthermore, the software can send a request to switch on or off the Whispergen. However, the controller built in the Gledhill decides whether the request can be honored or not.

This basic testbed is installed both in normal houses and in the laboratory. In the houses it replaces the conventional installation, in the laboratory some additional hard- and software is added. 
1) Additional hardware laboratory: In the laboratory configuration the Gledhill is connected to one radiator and a hot water tap. The radiator is represented by a forced heatexchanger on the roof of the test building.

A computer controlled relay card is used to switch on/off appliances and to generate the thermostat signal. Six relays switch the supply to outlets on or off, one relay is used to open and close a hot water tap valve and the last relay is used for the thermostat signal. We used a power analyzer to measure voltage, current, real and reactive load at once. This power analyzer can be connected to the PC via RS-232, so the measurement values can be logged.

All parts of the testbed can be connected with each other with normal $230 \mathrm{~V}$ plugs and outlets; a normal multiple socket connects all parts together.

For islanding tests and to use domestic electricity storage, battery equipment is added. For the battery equipment there are two important requirements:

- Charge and discharge the battery via one connection - Stabilize the $230 \mathrm{~V} / 50 \mathrm{~Hz}$

Battery solutions exist for both separate requirements, but as far as we know there is no battery solution commercially available that can stabilize the $230 \mathrm{~V} / 50 \mathrm{~Hz}$ while it is charging the battery. However, it should be possible to develop such a device. At the moment we are working together with a Dutch company, that has experience with battery solutions, on the development of such a battery solution. Until this solution is finished we use a battery, an inverter and a charger to charge the battery. The inverter inverts the $12 \mathrm{~V}=$ from the battery to the $230 \mathrm{~V} / 50 \mathrm{~Hz}$, supplies the shortage and stabilizes the $230 \mathrm{~V} / 50 \mathrm{~Hz}$. With this solution the stabilization requirement is met and the battery can be charged. To prevent that the battery is continuously charged, the charger is connected to one of the controlled outlets.

\section{B. Software prototype - Integration}

The hardware prototype is controlled via software. The core of the software delivers an interface to the hardware for other programs. This core part connects to HOMA software, determines the status of the Gledhill/Whispergen every minute and can send requests to start/stop the Whispergen. Furthermore, the controlling algorithms are implemented in this part of the software. The prediction information can be sent to a global controller via a TCP/IP connection. The local scheduler receives steering signals in the same way.

In the laboratory the core part of the software can also open/close the relays and can therefore manage appliances, the hot water tap valve, the central heating demand and the thermostat signal. Furthermore, it reads the values of the power analyzer every second. All information is timestamped and stored into a database.

For the laboratory setup a second software part is developed, the simulation part. This simulation part of the software can emulate a house by switching on/off appliances and by managing the heat demand (via the core). These two parts are connected via TCP/IP to allow them to run on different computers. Furthermore, a GUI in Python is built that can connect to the core to monitor the current situation and give commands manually (switch on/off appliances, the
Whispergen and the heat demand). A schematic representation of the hard- and software is given in Figure 3.

\section{RESUlts}

Three different scenarios are used to verify the methodology, the first two of them are backed by field tests. The first scenario is an islanded scenario to verify whether it is possible to create and maintain an islanded situation. The second scenario is a local scope scenario to verify whether the three step methodology is applicable in a real world situation. The last scenario is a simulation of a microgrid scope scenario to verify whether the methodology is applicable on a larger group and to verify the communication between global and local controller.

\section{A. Islanding}

Simulations presented in [27] showed that it is possible to supply most in an islanded situation with a Whispergen and a $1 \mathrm{kWh}$ battery, assuming that heat can be dumped. The algorithm described in the paper is a simplified version of the local controller described in this paper, but both controllers can reach the same results [30]. This shows the potential of islanding. Next, in [33] is showed that it is possible to start a Whispergen with a battery and run it islanded. Furthermore, it is possible to decide which appliance to supply and to charge the battery in an islanded situation. However, due to insufficient hardware it was not possible to test a complete islanded house (the combination).

With the inverter it is possible to start the Whispergen and to emulate an islanded house. Next to decide which appliances to supply and when to start the Whispergen, the control software must also decide when to switch on the battery charger. Furthermore, appliances need to be switched off when the Whispergen starts since it the Whispergen draws high startup currents. Finally, when the heat buffer is full it is not possible to start the Whispergen, so heat must be dumped then before the microCHP has to start. The simplified version of the control algorithm is used since it is easier to implement and monitor. An islanded situation is created during multiple hours with the testbed described in Section V. Three different appliances where used, the mircoCHP was switched on and off and battery was charged when there was a surplus. So, the control algorithm controlled the energy streams in an islanded house. However, it is rather hard to start the Whispergen with the current battery equipment. The high startup current lead to a voltage dip. The Whispergen protection against islanded operation (legislation) causes the machine switches off.

\section{B. Local scope}

In [30] we showed that peak shaving and shifting of demand in time using only a realtime scheduler is possible. In this test the prediction/planning/scheduling methodology is verified and also whether it is possible to actually switch on/off the appliance on the preferred times by the scheduler.

Using the simulator part of the software, a fixed heat demand profile is emulated. The heat demand of the previous day is used as prediction. Next, a planning is determined using a Dynamic Programming methodology. The objective 


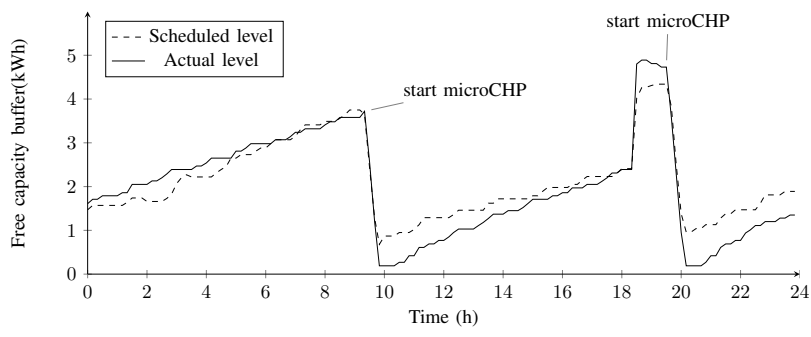

(a) Planned and actual free buffer capacity good heat prediction

Fig. 4. Results lab tests local planning and scheduling of a microCHP

is to shift production as much as possible to daylight hours (prevent noise at night). Furthermore, short runs are avoided (wearing of the machine), so only switch on signals are given (i.e. the generator runs until the buffer is filled). The planning is used as input for the scheduling algorithm. The planned and actual free capacity (inverse of the level) in the Gledhill for two different days is given in Figure 4. The runtime of the microCHP can be deducted from the free capacity, when the free capacity decreases the microCHP is running.

The heat demand prediction for the day in Figure 4(a) was accurate, but for the day in Figure 4(b) a peak in demand was predicted wrongly due to a delay in the hardware. Therefore, the planned and actual free capacity in Figure 4(a) are similar and, more important, the planned and actual runtimes of the microCHP are equal. Furthermore, the microCHP is started on initiative of the scheduler, since it was not required to switch on the microCHP due to the buffer level at $t=9.3$.

The wrong predicted peak in Figure 4(b) leads to a deviation in planned runtime of the microCHP of four hours. The planning was to run the microCHP until $t=10$, after the peak in demand. But, since the peak was too late, the microCHP had to be switched off because the heat buffer was filled $(t=8.5)$. The planning was to run until $t=10$, so there are steering signals and the microCHP switches on again at $t=9.7$. The heat demand between $t=10$ and $t=12$ is directly supplied while the microCHP was running (in contradiction to the planning). Therefore, it is not possible to switch on the microCHP at $t=14$.

The models and assumptions are accurate enough to determine a planning and it is possible to control the microCHP. However, when the heat demand deviates from the prediction, the planned and actual runtimes of the microCHP deviate as well.

\section{Microgrid scope}

The simulated scenario on a microgrid scope uses normal houses with a Whispergen/Gledhill combination, no electricity buffer and no controllable appliances. Therefore, the optimization potential is to shift the runtime of the microCHP in time. In the scenario four houses are simulated since heat demand data of four different houses during the winter season is available.

For a certain winter day the heat demand is predicted based on the historical data as described in [25] and with an improved version of this method. The predicted heat demand

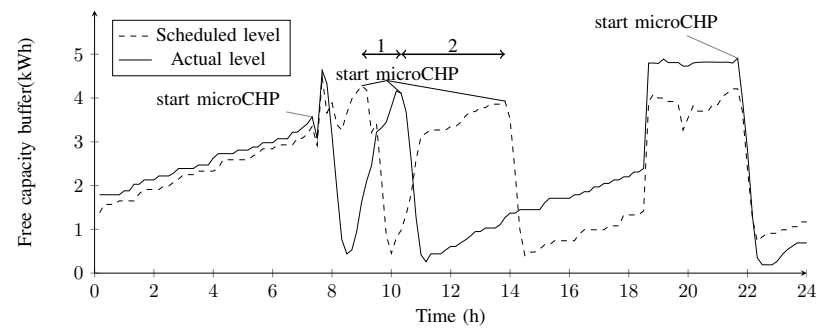

(b) Planned and actual free buffer capacity less good heat prediction 1) wrong predicted peak in demand 2) effect of wrong prediction

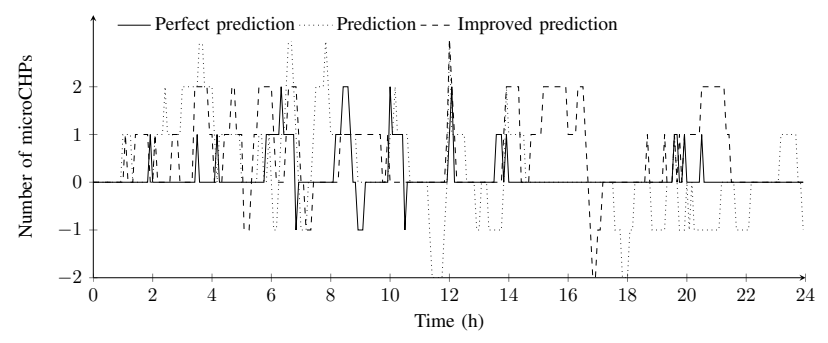

Fig. 5. Deviation of the number of microCHPs running on the planning for different prediction qualities

for every hour is sent to the global controller (24 values). Based on this prediction a planning a determined using the Linear Programming method described in [28]. The objective of this planning is to produce electricity when the electricity is expensive on the market. This planning is a sequence of on/off steering signals on a five minute time interval. The planning is used as input for the realtime scheduler, while the actual heat demand is used for the simulation. As reference, the actual heat demand is used as a perfect prediction.

The deviations on the planning for all three simulations is given in Figure 5. Remarkably there are also deviations on the planning with a perfect heat prediction. The models of the Whispergen and Gledhill used in the planning algorithm are less accurate than the models in the simulator and this causes the deviations. When the realistic heat demand prediction is used there is more deviation on the planning. It can be seen clearly that with a better prediction the deviation is lower. Most important of a microgrid or VPP scope optimization is to meet the predicted production, every imbalance is penalized. The imbalance for the perfect prediction is $4 \mathrm{kWh}$, for the prediction $16 \mathrm{kWh}$ and for the optimized prediction $15 \mathrm{kWh}$, with a total production of $50 \mathrm{kWh}$.

\section{DISCUSSION}

The methodology described meets almost all requirements deducted in Section IV-B. Only the requirement to be applicable for multiple scenarios is not met. The prediction only predicts heat demand and the global planning is tailored to combined heat/electricity generators and heatbuffers. Furthermore, the global controller needs knowledge of the configuration of every individual house leading to a lot of communication. Therefore, we are working on a generic way 
of expressing schedulingsfreedom and on versions of the prediction and planning that are more generic.

The first scenario showed that it is actual possible to create and maintain an islanded situation. Furthermore, the control algorithm is able to control the devices in a house: switch on/off a generator, switch on/off appliances and control the battery, even when the battery equipment is not optimal (charge $360 \mathrm{~W}$ or nothing). The assumption made for the simulations described in [27] seem to be valid. The second scenario showed that it is possible to determine a planning based on a prediction one day ahead. However, the prediction needs to be accurate, a wrongly predicted peak (for only a few minutes!) can have a severe impact on the runtime. Therefore, when the planning and actual situation deviate too much replanning is required. Replanning in the situation in Figure 4(b) at $t=8.5$ would have prevented the start at $t=9.7$ and the planning would have been followed better. The third scenario showed that it is possible to determine a planning for multiple houses. The communication between local and global part works fine, however too much specific information is required. Also in this situation is clear that the predictions need to be very accurate, however rescheduling can also in this situation decrease the amount of imbalance. Especially in a microgrid when a group of houses becomes self supporting or in a VPP when the optimizations are used for peak shaving the planning and actual import/export needs to be equal.

\section{REFERENCES}

[1] United States Department of Energy, "The micro-CHP technologies roadmap," Results of the Micro-CHP Technologies Roadmap Workshop December 2003

[2] D. Hammerstrom, R. Ambrosio, T. Carlon, J. DeSteese, G. Horst, and R. Kajfasz, "Pacific northwest gridwise testbed demonstration projects, part i and ii," Pacific Northwest National Laboratory, July 2007.

[3] E. Furling, M. Piemontesi, P. Prasad, and D. Sukumar, "Advances in energy storage techniques for critical power systems," in The Battcon 2002 proceedings, 2002.

[4] A. Peacock and M. Newborough, "Controlling micro-chp systems to modulate electrical load profiles," Energy, vol. 32, no. 7, pp. 1093-1103, July 2007.

[5] M. Newborough and P. Augood, "Demand-side management opportunities for the uk domestic sector," in IEE Proceedings Generation, Transmission and Distribution, vol. 3, May 1999, pp. 283-293.

[6] C. Block, D. Neumann, and C. Weinhardt, "A market mechanism for energy allocation in micro-chp grids," in 41st Hawaii International Conference on System Sciences, Jan 2008, pp. 172-180.

[7] J. Scott, P. Vaessen, and F. Verheij, "Reflections on smart grids for the future," Dutch Ministry of Economic Affairs, Apr 2008

[8] "Distributed generation in liberalised electricity markets," 2002

[9] D. S., N. M., and P. S. D., "Rescheduling electricity demands in domestic buildings," Applied Energy, vol. 44, no. 1, pp. 1-62, 1993.

[10] S. Abu-sharkh, R. Arnold, J. Kohler, R. Li, T. Markvart, J. Ross, K. Steemers, P. Wilson, and R. Yao, "Can microgrids make a major contribution to uk energy supply?" Renewable and Sustainable Energy Reviews, vol. 10, no. 2, pp. 78-127, Sept 2004.

[11] A. Azmy and I. Erlich, "Impact of distributed generation on the stability of electrical power system," in Power Engineering Society General Meeting, 2005. IEEE, June 2005, pp. 1056-1063 Vol. 2.

[12] R. Morgan, J. Devriendt, and B. Flint, "Microchp a mass market opportunity?" Sustainability: Microgeneration, 2006.

[13] L. Costa, F. Bourry, J. Juban, and G. Kariniotakis, "Management of energy storage coordinated with wind power under electricity marke conditions," in Probabilistic Methods Applied to Power Systems, 2008. PMAPS '08. Proceedings of the 10th International Conference on, May 2008, pp. $1-8$

[14] N. Lu and D. Hammerstrom, "Design considerations for frequency responsive grid friendlytm appliances," in Transmission and Distribution Conference and Exhibition, 2005/2006 IEEE PES, May 2006, pp. 647652
[15] N. Lu and T. Nguyen, "Grid friendlytm appliances - load-side solution for congestion management," in Transmission and Distribution Conference and Exhibition, 2005/2006 IEEE PES, May 2006, pp. 1269-1273.

[16] J. Oyarzabal, J. Jimeno, J. Ruela, A. Englar, and C. Hardt, "Agent based micro grid management systems," in Internation conference on Future Power Systems 2005. IEEE, Nov 2005, pp. 6-11.

[17] J. Kok, C. Warmer, and I. Kamphuis, "Powermatcher: Multiagent control in the electricity infrastructure," in 4th international joint conference on Autonomous agents and multiagent systems. ACM, Jul 2005, pp. 75-82.

[18] M. Hommelberg, B. van der Velde, C. Warmer, I. Kamphuis, and J. Kok, "A novel architecture for real-time operation of multi-agent based coordination of demand and supply," in Power and Energy Society General Meeting - Conversion and Delivery of Electrical Energy in the 21st Century, 2008 IEEE, July 2008, pp. 1-5.

[19] C. Warmer, M. Hommelberg, B. Roossien, J. Kok, and J. Turkstra, "A field test using agents for coordination of residential micro-chp," in Intelligent Systems Applications to Power Systems, 2007. ISAP 2007. International Conference on, Nov. 2007, pp. 1-4.

[20] A. Dimeas and N. Hatziargyriou, "Agent based control of virtual power plants," in Intelligent Systems Applications to Power Systems, 2007. ISAP 2007. International Conference on, Nov. 2007, pp. 1-6.

[21] R. Caldon, A. Patria, and R. Turri, "Optimisation algorithm for a virtual power plant operation," in Universities Power Engineering Conference, 2004. UPEC 2004. 39th International, vol. 3, Sept. 2004, pp. 1058-1062 vol. 2.

[22] E. Handschin and F. Uphaus, "Simulation system for the coordination of decentralized energy conversion plants on basis of a distributed data base system," in Power Tech, 2005 IEEE Russia, June 2005, pp. 1-6.

[23] L. Costa and G. Kariniotakis, "A stochastic dynamic programming model for optimal use of local energy resources in a market environment," in Power Tech, 2007 IEEE Lausanne, July 2007, pp. 449-454.

[24] J. V. Ringwood, D. Bofelli, and F. T. Murray, "Forecasting electricity demand on short, medium and long time scales using neural networks," Journal of Intelligent and Robotic Systems, vol. 31, no. 1-3, pp. 129147, december 2004.

[25] V. Bakker, A. Molderink, J. Hurink, and G. Smit, "Domestic heat demand prediction using neural networks," in 19th International Conference on System Engineering. IEEE, 2008, pp. 389-403.

[26] A. Molderink, M. Bosman, V. Bakker, J. Hurink, and G. Smit, "Simulating the effect on the energy efficiency of smart grid technologies (accepted)," in Winter Simulation Conference 2009. IEEE, 2009.

[27] A. Molderink, V. Bakker, J. Hurink, and G. Smit, "Algorithms for balancing demand-side load and micro-generation in islanded operation," in 19th International Conference on System Engineering. IEEE, 2008, pp. 389-403.

[28] M. Bosman, V. Bakker, A. Molderink, J. Hurink, and G. Smit, "The microchp scheduling problem," in Second Global Conference on Power Control and Optimization. IEEE, 2009.

[29] - "On the microchp scheduling problem (accepted)," in Third Global Conference on Power Control and Optimization. IEEE, 2010.

[30] A. Molderink, V. Bakker, M. Bosman, J. Hurink, and G. Smit, "Domestic energy management methodology for optimizing efficiency in smart grids," in IEEE conference on Power Technology. IEEE, 2009.

[31] http://www.whispergen.com.

[32] http://www.gledhill.net/.

[33] A. Molderink, M. Bosman, V. Bakker, J. Hurink, and G. Smit, "Hardand software implementation and verication of an islanded house prototype," in International Conference on System Engineering. IEEE, 2009.

\section{BIOPGRAPHIES}

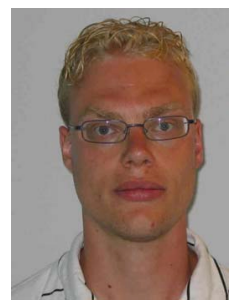

Albert Molderink was born in Heerenveen (The Netherlands) in 1983. He received his B.Sc and M.Sc. degree in Computer Science from the University of Twente, Enschede, The Netherlands, in respectively 2004 and 2007. In addition, he received a Electrical Engineering minor certificate. When he completed his study he started working towards a $\mathrm{Ph} . \mathrm{D}$. degree at the University of Twente under supervision of Prof. dr. ir. G.J.M. Smit.He is working in a research group that investigates the possibilities of increasing energy efficiency using embedded control, mainly via optimization and control algorithms. His research focus is on algorithms to optimize energy streams within a house. 